\title{
Optimization of the readout of microdrum optomechanical
}

\section{resonators}

Valerio Pinia,c), Daniel Ramos ${ }^{\mathrm{a}, \mathrm{c}) *}$, Carmen M. Dominguez ${ }^{\mathrm{a}}$, Jose Jaime Ruz ${ }^{\mathrm{a}}$, Oscar Malvar $^{\mathrm{a}}$, Priscila M. Kosaka ${ }^{\mathrm{a}}$, Zachary J. Davis ${ }^{\mathrm{b}}$, Javier Tamayo ${ }^{\mathrm{a}}$ and Montserrat Calleja $^{\mathrm{a}}$

a Bionanomechanics Lab, Instituto de Micro y Nanotecnología, IMN-CNM (CSIC), Isaac Newton 8 (PTM), E-28760 Tres Cantos, Madrid, Spain. ${ }^{b}$ Danish Technological Institute, Gregersensvej 1, 2630 Taastrup, Denmark.

*corresponding author: daniel.ramos@csic.es

c) V. Pini and D. Ramos contributed equally to this work

Free-standing dielectric structures with a substrate underneath, as the SiN microdrums studied here, are the basis of optomechanical devices. Optimization of the optomechanical coupling is demanding in order to enhance the performance of these devices. The optomechanical coupling in the case of drum resonators critically depends on the thickness of the drum, the surface stress, the cavity length and the wavelength. Here, we develop a methodology that combines spatially multiplexed microspectrophotometry, optical modelling of the cavity and finite element method simulation of the mechanical eigenmodes that enables obtaining the properties of the optomechanical cavity and predicting the wavelength that optimizes the optomechanical coupling. By choosing this illumination wavelength we are able to spatially resolve the thermal motion of the microdrums up to the fourth mechanical mode $\sim 20 \mathrm{MHz}$. The presented study opens the door for further optimization pathways for optomechanical detection and actuation.

Last decade has witnessed the advent of nanomechanical devices as a new platform for sensing applications in many diverse fields like physical ${ }^{1,2}$, biological $^{3-5}$ or chemical applications ${ }^{6}$. In that sense, the fabrication tools developed by the semiconductor industry have paved the way for the development of different kinds of devices ranging from 1D-like resonators such as silicon nanowires ${ }^{7,8}$ or carbon nanotubes $^{9}$ to $2 \mathrm{D}$ structures like suspended graphene membranes ${ }^{10,11}$. The everincreasing control reached in these nanofabrication techniques has allowed remarkable 
milestones such as yoctogram range mass resolution ${ }^{12}$ or single spin moment detection ${ }^{2}$. In these unprecedented advances, the use of new materials has played an important role, being the carbon nanotubes and graphene sheets the most promising candidates for 1D and $2 \mathrm{D}$ nanomechanical resonators, respectively. However, the bottom up fabrication techniques present counteracts derived not only from the quality of the structural material but also from the necessary integration. Typically, the whole electrical circuitry of the detection device must be patterned around the randomly deposited element, resulting in a costly and time-consuming process; which constitutes the main reason why top-down lithographic techniques are commonly employed these days, with silicon nitride ${ }^{13,14}$ and $\mathrm{MoS}_{2}$ as prominent materials ${ }^{15}$.

As the nanofabrication techniques shrink down the size of the resonators, the read-out process becomes more complicated. Optical methods ${ }^{16,17}$ are usually employed due to their versatility and, in principle, non-physically limited bandwidth, which is important when dealing with small oscillators with natural resonance frequencies ${ }^{18}$ lying in the verge of $10^{6}$ to $10^{9} \mathrm{~Hz}$. However, as the lateral size of the resonators approaches the illuminating wavelength, interplay between the mechanical object and the light, or optical back-action ${ }^{19}$, arises, resulting in a new branch in the community known as optomechanics ${ }^{20,21}$.

In this work, we present a thorough optmomechanical characterization of suspended silicon nitride membranes; the accurate measurement of the geometric and optical properties of the devices under study will allow to determine a priori the theoretical mechanical sensitivity and, therefore, the best design strategy to optimize the interferometric transduction mechanism. The optical characterization consists of the analysis of the reflectivity as a function of the illuminating wavelength; this measurement allows us accurate characterization of the thickness and the refractive index of the suspended structure. By studying the reflectivity change, we are able to calculate the mechanical displacement sensitivity of the system, which depends on the illuminating wavelength; therefore, we can anticipate that the best displacement measurement is obtained by using a red laser $(632.8 \mathrm{~nm})$ instead of a green laser (542 $\mathrm{nm})$. This theoretical prediction was experimentally demonstrated; however, the theoretically expected mechanical resonance frequency is one order of magnitude smaller than the measured one. This discrepancy is explained in terms of the initial stress accumulated in the membrane during the fabrication process. Due to the geometrical constrains, this stress cannot be released and the membrane consequently 
buckles. The buckling was measured by using AFM, giving us a static deformation of about $2.3 \mathrm{~nm}$. Finite element simulations were employed to simulate a tense structure ${ }^{22}$, giving a resulting value for the stress of $927 \mathrm{MPa}$. The resonance frequencies calculated by taking into account this value agree the experimental values for the first four mechanical modes.

The experimental set-up was made by means of a homemade hybrid interferometer, represented in a block diagram in Fig. 1a. By means of flip-mounts, we can use two different configurations for both the optical and mechanical characterization by choosing between two different illuminating sources: a Xe lamp for the optical characterization and a laser unit (red or green depending on the application) for the mechanical characterization. In order to carry out the optical characterization, the illumination beam coming out from the Xe lamp is directed into a monochromator that disperses the light into its constituent wavelengths. A narrow band of the dispersed spectrum passing through the exit slit of the monochromator is then collimated by a series of lenses and focused onto the testing sample by a microscope objective. The light reflected from the surface is then collected by the same microscope objective and forms an image on the surface of a CCD camera passing through a non-polarized beam splitter.

The nanofabrication of arrays of $100 \mathrm{~nm}$ thick silicon-nitride membranes process is performed by using standard cleanroom procedures. First, thermal oxidation was performed growing $1370 \mathrm{~nm}$ thick thermal oxide on a (100) silicon 6" wafer. The oxide was then controllably etched down to a $1270 \mathrm{~nm}$ thickness by wet chemical etching using BHF (Buffered hydrofluoric acid). Then, a $220 \mathrm{~nm}$ layer of silicon nitride was deposited with LPCVD (Low Pressure Chemical Vapor Deposition). The deposited layer of silicon nitride was thicker than the final desired one to compensate the nonnegligible etch rate of the hydrofluoric acid on the silicon nitride, which was calibrated prior to the device nanofabrication. After the oxide and silicon nitride deposition steps, contact UV lithography was performed following reactive ion etching (RIE) to drill the holes in the top silicon nitride layer. Finally, the plate was released by controlled etching of the silicon oxide release layer through the holes using wet hydrofluoric acid. As anticipated the wet HF etch also etches the silicon nitride plate to a thickness of approximately $100 \mathrm{~nm}$. 
Inset in Fig. 1b shows a topographic Atomic Force Microscopy (AFM) image of the membrane resonator (tapping mode, cantilever from Nanosensors PPP-NCH, spring constant $\sim 42 \mathrm{~N} / \mathrm{m}$, resonance frequency $\sim 330 \mathrm{kHz}$ ). The membrane is buckled up due to the accumulated stress during the silicon nitride deposition process. Since all the edges of the membrane are clamped, there is no way to release the initial stress, and the structure is buckled. The AFM characterization reveals a static deformation of $2.3 \mathrm{~nm}$ upwards at the center of the membrane, indicating a non-released stress of $927 \mathrm{MPa}$ according to the FEM parametric study performed by sweeping the stress of the layer to fit the measured deflection.

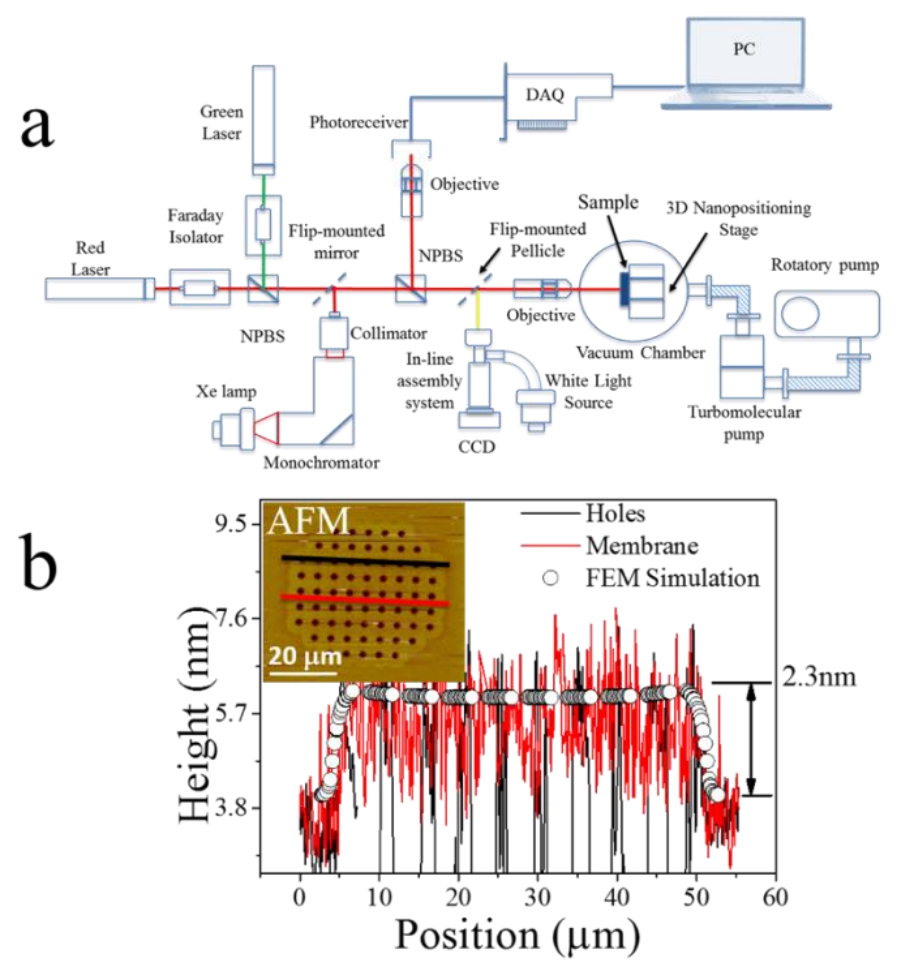

Fig. 1.a. Schematics of the experimental set-up used for the optical and mechanical characterization of the silicon membranes. b. The black curve shows the spatial profile of the topography measured with AFM over a line of holes (black line drawn in the inset); whereas the red line shows the profile avoiding the holes, along the red line drawn in the inset. The open symbols show the static deformation numerically calculated with FEM by considering an initial tensile stress of $927 \mathrm{MPa}$.

Therefore, there are two different regions to perform the reflectivity study: the holey membrane and the silicon nitride continuous layer. The difference in between them is basically the materials forming the Fabry-Perot optical cavity. In the continuous 
silicon nitride layer, black dot in the optical micrograph of the inset in Fig. 2a, the two Fabry-Perot cavities are composed by the silicon nitride layer and the silicon dioxide underneath, whereas in the membrane region, red dot in the inset of Fig. 2a, the double Fabry-Perot cavity is composed by the holey silicon nitride layer and the air region. The measured reflectivity in both regions are plotted in Fig. 2a, being the solid black line the experimental reflectivity measurements on the continuous silicon nitride layer and the solid red line the values on the released membrane. Both measurements where normalized by the bulk silicon reflectivity, which removes the lamp intensity dependency on the selected wavelength, by using the recently developed SMMS technique $^{23}$. Therefore, the measurement represents the relative change in percentage of the sample reflectivity compared with bulk silicon.

Previous studies in literature ${ }^{24-26}$ show that the overall reflectivity depends not only on the illumination wavelength but also on the numerical aperture of the objective, the light polarization and the spatial coherence of the light source. In Fig. 2b, we have calculated the reflectivity spectra of the silicon nitride membrane for different numerical apertures from 0 to 0.65 by considering the Xe lamp as an incoherent unpolarized light source. The use of a microscope objective with a finite numerical aperture NA produces a light cone illumination with an acceptance angle of $\theta_{N A}=\sin ^{-1}(N A)$. Since the Fabry-Perot interferences responsible for the detection depend on the length of the optical path, the simultaneous illumination of light beams with different incidence angles produces an overall reflectivity given by the superposition of multiple optical cavities, that for an uncoherent light source is given by

$$
R_{w, \text { uncoh }}=\frac{1}{\theta_{N A}} \int_{0}^{\theta_{N A}}\left|r_{w}(\theta)\right|^{2} d \theta
$$

where the subscript $w$ determines the light polarization state and $r_{w}(\theta)$ is the complex amplitude of the reflected wave ${ }^{24}$ at the incidence angle $\theta$.

These theoretical predictions were experimentally demonstrated by measuring the reflectivity spectra of the silicon nitride membranes at different numerical apertures and by illuminating the sample surface with an unpolarized Xe lamp, Fig. 2b. The numerical aperture of the illuminating light was experimentally controlled by using optical objectives with different magnification (10X, 20X and 50X) and by adjusting the aperture stop of the optical microscope. Hence, the effective numerical aperture is given 
by a combination of the numerical aperture of the objective and the aperture stop of the optical setup. Additionally, in the experiments it was found a progressive attenuation of the reflectivity modulation by increasing the numerical aperture.

The reflectivity of a Fabry-Perot cavity can be simply modeled by using the well-known Fresnel equations ${ }^{24}$,

$$
R=\left|\frac{a_{-}+b_{+}}{a_{+}+b_{-}}\right|^{2},
$$

where $a_{\mp}$ and $b_{ \pm}$are given by

$$
\begin{aligned}
& a_{\mp}=\left[e^{-\phi_{1}+\phi_{2}}\left(n_{0} \mp n_{1}\right)\left(n_{1}-n_{2}\right)+e^{\phi_{1}+\phi_{2}}\left(n_{0} \pm n_{1}\right)\left(n_{1}+n_{2}\right)\right]\left(n_{2}-n_{3}\right) \\
& b_{ \pm}=\left[e^{\phi_{1}-\phi_{2}}\left(n_{0} \pm n_{1}\right)\left(n_{1}-n_{2}\right)+e^{-\phi_{1}-\phi_{2}}\left(n_{0} \mp n_{1}\right)\left(n_{1}+n_{2}\right)\right]\left(n_{2}+n_{3}\right) ;
\end{aligned}
$$

the subscripts correspond to the order number of the layer considered, being 0 the top layer and 3 the bottom layer. The phase $\phi_{m}=2 i d_{m} n_{m} \pi / \lambda$ with $m=1,2$ is the optical path, being $d_{m}$ the thickness of the layer $m$ and $n_{m}$ its complex refractive index. In order to account for the wavelength dependency of the lamp intensity, this theoretical reflectivity needs to be normalized to the reflectivity of the silicon substrate $R_{\text {ref }}=$ $\left|\frac{1-\mathrm{n}_{3}}{1+\mathrm{n}_{3}}\right|^{2}$, obtaining the following expression $\bar{R}=\frac{R-R_{\mathrm{ref}}}{R_{\mathrm{ref}}}$.

Therefore, by fitting the reflectivity signal at the silicon nitride layer to the Fresnel reflectivity equations, it is possible to extract the values of the layer thicknesses, being $(142 \pm 1) \mathrm{nm}$ for the silicon nitride and $(1271 \pm 1) \mathrm{nm}$ for the silicon dioxide. Imposed by fabrication conditions, the thickness of the silicon dioxide is the same as than the air layer under the suspended membrane; hence, we can use the calculated thicknesses to estimate the refractive index of the membrane. By fitting with the appropriate material properties, refractive index of the membrane material results in a smaller value than the bulk silicon nitride refractive index, $n^{\text {membrane }}=0.94 n^{\operatorname{SiN}}$. We can explain this discrepancy by simply attending at geometrical considerations. The whole membrane presents 69 holes with a radius of $750 \mathrm{~nm}$, therefore, the area of the actual membrane is $6 \%$ smaller than the area of a hole-free membrane, resembling the results of the calculated refractive index.

Once we have established the actual thickness and refractive index of the membrane, we can make an estimation of the displacement sensitivity within an interferometric measurement, which is given by $\partial R^{\text {membrane }} / \partial \lambda$, blue curve in Fig. $2 \mathrm{a}$. As we can see in this plot, the displacement sensitivity largely depends on the used 
wavelength. The reason behind this is the same as for the oscillatory behavior of the reflectivity, red curve in Fig. 2a: the constructive and destructive interferences in the Fabry-Perot optical cavities. In this sense, the displacement sensitivity is maximum around $650 \mathrm{~nm}$ and presents a local minimum around $550 \mathrm{~nm}$, besides the points of null sensitivities distributed along the entire spectrum. It is possible to experimentally demonstrate this wavelength sensitivity dependency by simply replacing the illuminating source by a laser unit, at red (Thorlabs Inc, $632.8 \mathrm{~nm}, 5 \mathrm{~mW}$ ) or green (Thorlabs Inc, $542 \mathrm{~nm}, 3 \mathrm{~mW}$ ) wavelengths. In order to compare the mechanical amplitude measured with both lasers, a variable neutral density filter is used to adjust the laser power of both sources to be exactly the same at the sample plane, of about 0.1 $\mathrm{mW}$.
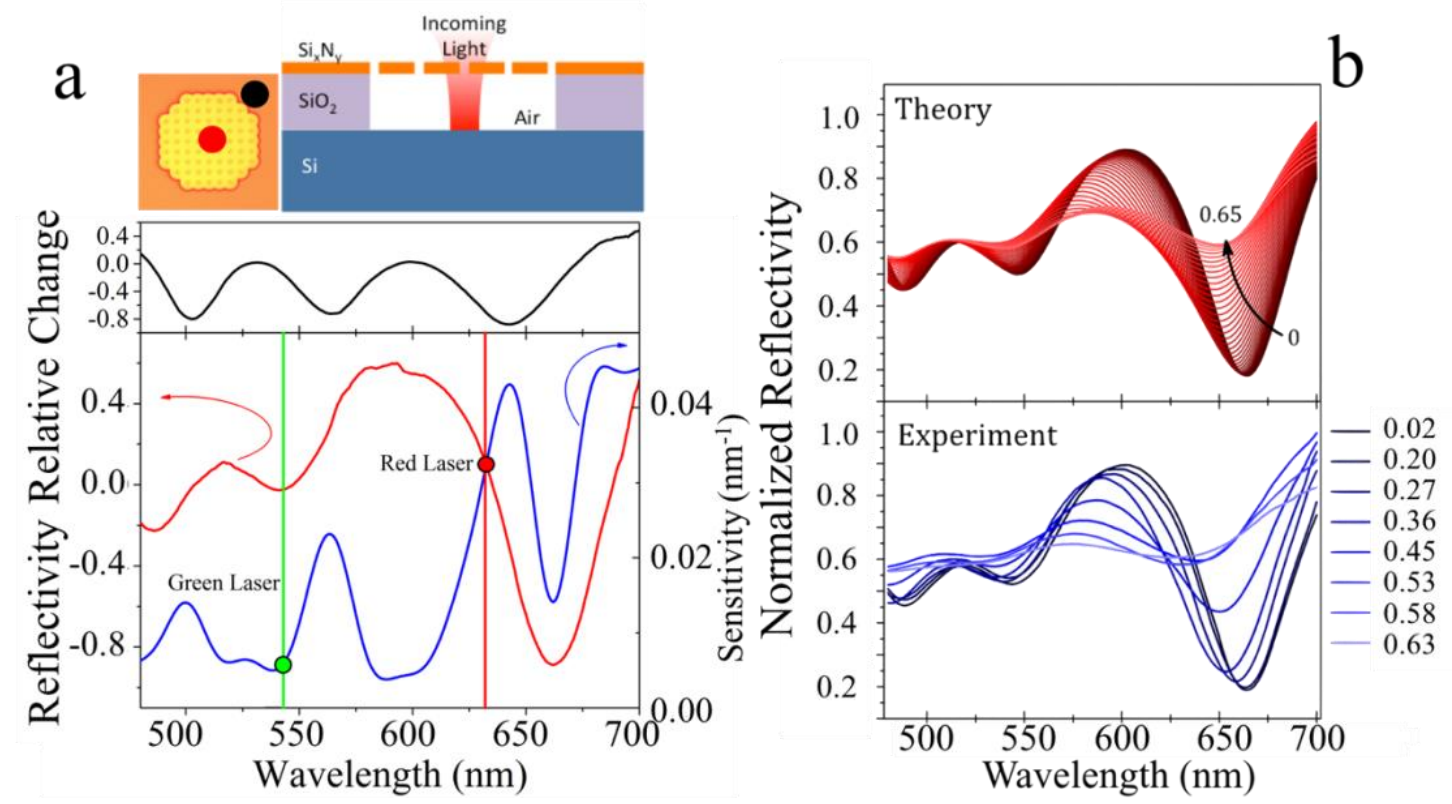

Fig. 2.a. Schematic drawing of the suspended silicon-nitride membrane. Experimental measurements of the normalized reflectivity measured outside and inside the suspended plate (black and red solid lines respectively) as a function of the illumination wavelength. The dots in the optical image show the measurement places. The solid blue line represents the sensitivity. b. up. Theoretical spectra of the normalized-reflectivity calculated for different numerical apertures and obtained for a spatially incoherent and unpolarized light source. down. Experimental normalized reflectivity spectra obtained for different numerical apertures by illuminating the sample with an unpolarized Xenon lamp.

Fig. 3a shows the experimental measurement of the fundamental mechanical mode, whose resonance frequency is of $8.6 \mathrm{MHz}$, by using both lasers. The red curve 
represents the measurement using the red laser $(632.8 \mathrm{~nm})$, whereas the green curve corresponds to the green laser (542 nm). As can be clearly seen, the measured amplitude with the green laser is barely visible, being almost completely covered by the ground floor noise. However, the signal to noise ratio measured by the red laser is approximately 2, corresponding to a properly resolved Lorentzian peak. By using the calculated data of the silicon nitride thickness, and the lateral size of the membrane and holes, the theoretical resonance frequency of the fundamental mechanical mode is 1.07 $\mathrm{MHz}$, which clearly differs from the measured one in Fig. 3a by almost one order of magnitude. As it was previously described, the difference comes from the stress in the silicon nitride layer of $927 \mathrm{MPa}$.

The optimization of the interferometric detection mechanism showed before, guarantees the measurement of tiny thermal vibrations with higher signal to noise ratio and, thus, the possibility to observe interesting features in the spatial mapping by a $2 \mathrm{D}$ scanning of the sample. Fig. 3a shows the spatial profile of the vibration amplitude measured across the membrane avoiding the holes. The overall spatial profile (red solid line in Fig. 3a) is given by the convolution of the fundamental vibration mode (black dashed line) with a periodical signal whose spatial periodicity is equal to the distance between the holes $(\cong 5 \mu \mathrm{m})$. This periodical signal is produced by the presence of some nanofabrication defects of the device. The reflectivity modulation arises by the presence of some oxide residues that were not fully etched from the underneath silicon substrate. In fact, as silicon oxide below the holes is etched faster than the oxide situated between the etch holes, this results in the formation of a square grid of silicon oxide crests.

In order to corroborate this hypothesis, we have removed the membrane by exfoliating the sample with a tape stripping process. White light interferometry carried out with a commercial setup (Wyko NT9100, Veeco) confirmed the presence of etching oxide residues (Fig. 3b) in between the holes. The height of the oxide residues measured by white light interferometry is of about $25 \mathrm{~nm}$, with a spatial periodicity of about $5 \mu \mathrm{m}$, as can be seen from lower chart in Fig. $3 b$. 

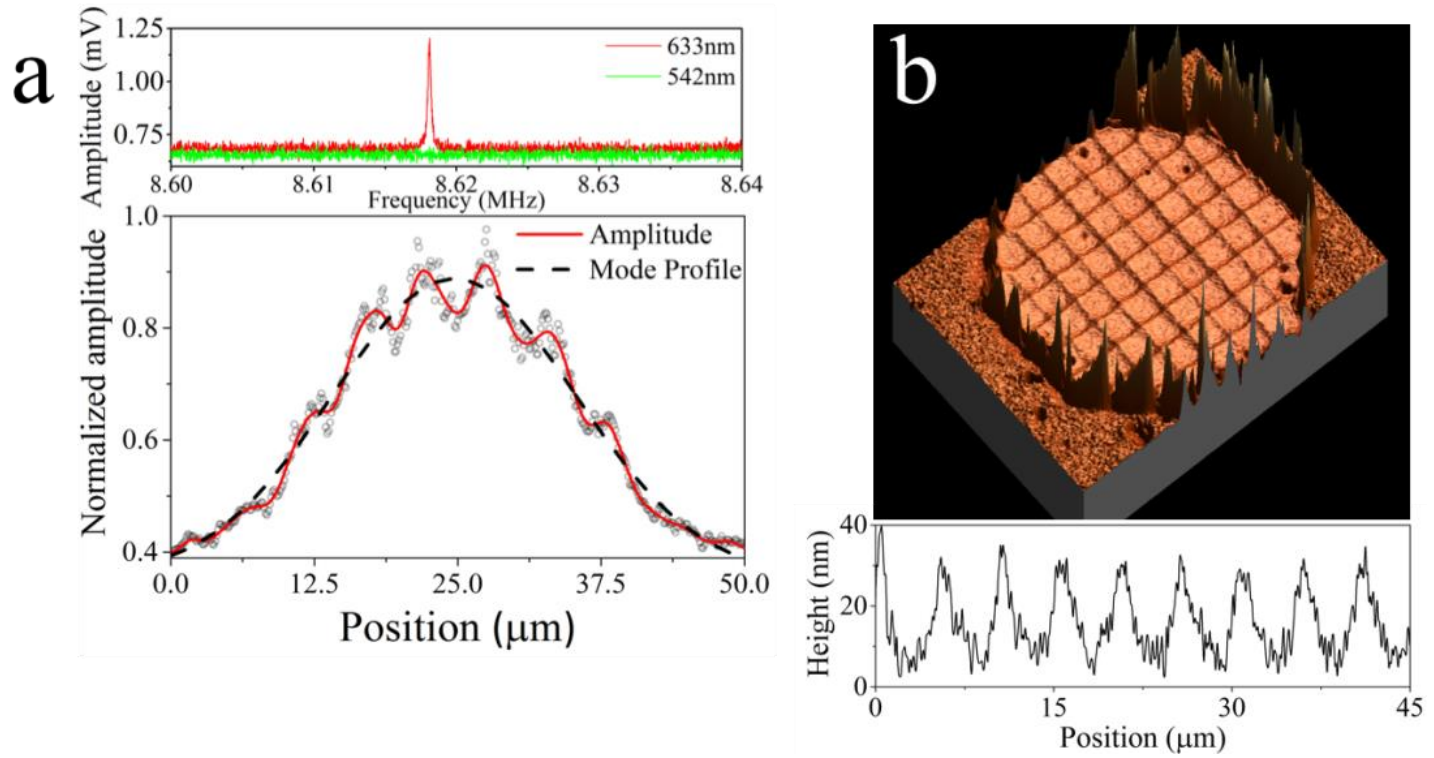

Fig. 3.a. up. Thermal spectra of the fundamental mechanical vibration mode measured by using a $632.8 \mathrm{~nm}$ laser (red line) and a $542 \mathrm{~nm}$ laser (green line). The spectrum measured with the green light is barely visible, as expected. down. Spatial profile of the fundamental mechanical mode (red line). The theoretically predicted mode profile is shown as black dashed line. b. up. Experimental topography measurement of the surface below the membrane after the exfoliation of the suspended SiN layer. Experiments were carried out with a commercial white light interferometer (Veeco). down. Experimental profile measured by using the white light interferometer.

The resonant frequencies of tensile circular membranes with diameter $\mathrm{D}$ can be modeled by simply using classical elasticity theory, following this expression ${ }^{27,28}$

$$
f=\xi_{n, m} \frac{1}{\pi D} \sqrt{\frac{\sigma}{\rho}}
$$

where $\xi_{n, m}$ is the $\mathrm{m}^{\text {th }}$ zero of Bessel function $J_{n}(x)$ for the same $(\mathrm{m}, \mathrm{n})$ indices referred above, $\sigma$ is the stress and $\rho$ the mass density. By introducing the calculated value of the stress in this equation, besides the silicon nitride mass density taking into account the holes, $\rho^{\text {membrane }}=0.94 \rho^{\operatorname{SiN}}$, we have calculated the frequencies for the first four mechanical modes: $f_{(1,0)}^{\text {model }}=8.6 \mathrm{MHz}, f_{(1,1)}^{\text {model }}=13.7 \mathrm{MHz}, f_{(1,2)}^{\text {model }}=18.4 \mathrm{MHz}$, and $f_{(2,0)}^{\text {model }}=19.8 \mathrm{MHz}$; which perfectly match the calculated values by using finite element method. The interferometric technique described above allows to measure the actual mechanical displacement 2D maps by simply scanning the sample, Fig. 4a-d, experimental measurements show a very good agreement with both the finite element method and the elasticity model. 


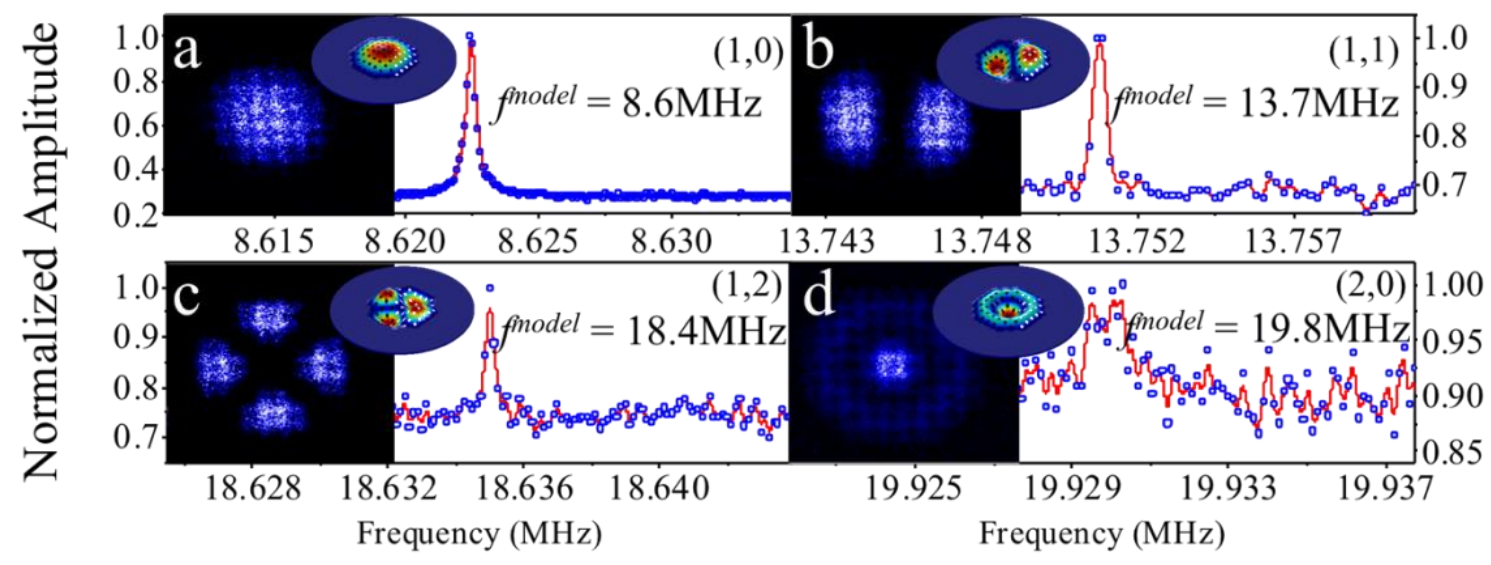

Fig. 4. a-d. Experimental measurements of the first four mechanical modes. The colour contour plots show the amplitude of the mode measured by scanning the sample. The FEM simulations of the amplitudes are also plotted for comparison.

In conclusion, we have presented a method for the mechano-optical characterization of freestanding membranes sustaining an optical cavity. The optical characterization of the reflectivity spectrum allows accurately measuring the thicknesses of the different structural layers of the device. We have used this thickness characterization to theoretically calculate and experimentally demonstrate the displacement sensitivity of the system, which results to be at least one order of magnitude larger when using a red laser compared to the use of a green laser. The discrepancy between the measured mechanical resonance frequency and the calculated one by FEM was attributed to the tensile stress in the silicon nitride layer. This stress was calculated by measuring the membrane buckling with the AFM and fitting the resulting value with the FEM static deflection simulations. This stress shifts the fundamental resonance to higher frequencies from $1 \mathrm{MHz}$ to $8.6 \mathrm{MHz}$, being also confirmed by classical elasticity theory.

\section{Acknowledgement}

This work was supported by the Spanish Science Ministry (MINECO) through project MAT2015-66904-R and by the European Union's Horizon 2020 research and innovation program under grant agreement No 731868-VIRUSCAN. D.R. acknowledges the RyC fellowship supported by MINECO. 


\section{References}

W. Lee et al., Proceedings of the National Academy of Sciences 106 (36), 15225 (2009).

D. Rugar et al., Nature 430 (6997), 329 (2004).

J. Mertens et al., Nat Nanotechnol 3 (5), 301 (2008).

P. M. Kosaka et al., Nat Nanotechnol 9 (12), 1047 (2014).

C. M. Domínguez et al., Scientific Reports 7 (1), 536 (2017).

P. S. Waggoner et al., Lab on a Chip 7 (10), 1238 (2007).

E. Gil-Santos et al., Nat Nanotechnol 5 (9), 641 (2010).

D. Ramos et al., Sci Rep 3, 3445 (2013).

JensenK et al., Nat Nano 3 (9), 533 (2008).

J. S. Bunch et al., Nano Letters 8 (8), 2458 (2008).

V. P. Adiga et al., Applied Physics Letters 103 (14), 143103 (2013).

J. Chaste et al., Nat Nanotechnol 7 (5), 301 (2012).

A. M. Jayich et al., New Journal of Physics 14 (11), 115018 (2012).

D. J. Wilson et al., Physical Review Letters 103 (20), 207204 (2009).

A. Castellanos-Gomez et al., Advanced Materials 24 (6), 772 (2012).

K. L. Ekinci, Small 1 (8-9), 786 (2005).

T. Kouh et al., Applied Physics Letters 87 (11), 113112 (2005).

X. M. Henry Huang et al., Nature 421 (6922), 496 (2003).

E. Gil-Santos et al., New Journal of Physics 15 (3), 035001 (2013).

M. Aspelmeyer et al., Reviews of Modern Physics 86 (4), 1391 (2014).

R. A. Norte et al., Physical Review Letters 116 (14), 147202 (2016).

D. R. Southworth et al., Physical Review Letters 102 (22), 225503 (2009).

V. Pini et al., Scientific Reports 6, 22836 (2016).

M. Born et al., Principles of Optics: Electromagnetic Theory of Propagation, Interference and Diffraction of Light. (Cambridge University Press, 2000).

G. W. Stroke, An Introduction to Coherent Optics and Holography. (Academic Press, 1971).

M. Sarrazin et al., Optics Express 21 (S4), A616 (2013).

K. F. Graff, Wave Motion in Elastic Solids. (Dover Publications, 2012).

A. Borrielli et al., Physical Review B 94 (12), 121403 (2016). 